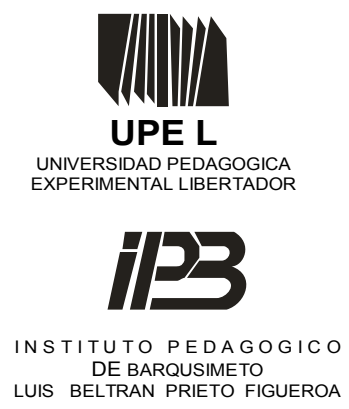

\title{
REVISTA
}

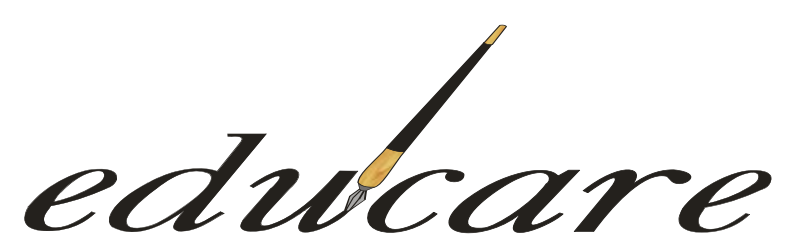

Órgano Divulgativo de la Subdirección de Investigación y Postgrado del Instituto Pedagógico de Barquisimeto "Luis Beltrán Prieto Figueroa”

BARQUISIMETO - EDO. LARA - VENEZUELA

NUEVA ETAPA

FORMATO ELECTRÒNICO

DEPOSITO LEGAL:

PPI2O 1002LA3674

VOLUMEN 19 №

ISSN: 2244-7296

\author{
SEPTIEMBRE - DICIEMBRE 2015
}

\section{REPENSAR LA ESCUELA: MI CONFESIÓN, UN TEXTO, LOS ESTUDIANTES OTROS TEXTOS}

RETHINKING SCHOOL: MY CONFESSION, A TEXT, STUDENTS OTHER TEXTS

\author{
CARLOS MOSQUERA* \\ INGRID RONDÓN
}

*Secretaría departamental de Educación, ANTIOQUia 


\section{REPENSAR LA ESCUELA: MI CONFESIÓN, UN TEXTO, LOS ESTUDIANTES OTROS TEXTOS}

\section{RETHINKING SCHOOL: MY CONFESSION, A TEXT, STUDENTS OTHER TEXTS}

\begin{tabular}{|c|c|}
\hline$\underline{\text { ENSAYO }}$ & $\begin{array}{r}\text { Carlos Mosquera* } \\
\text { Ingrid Rondòn** } \\
\text { Secretaría Departamental de Educación - } \\
\text { Antioquia }\end{array}$ \\
\hline Recibido:02/09/2015 & Aceptado: 27/11/2015 \\
\hline RESUMEN & ABSTRACT \\
\hline $\begin{array}{l}\text { El presente ensayo tiene como propósito dar a } \\
\text { conocer el relato docente, como posibles } \\
\text { dispositivos pedagógicos para mejorar la práctica } \\
\text { docente. La estrategia metodológica consistió en } \\
\text { realizar un análisis documental a partir de algunos } \\
\text { autores que abordan la temática, esto acompañado } \\
\text { de narrativas de maestros en el entorno escolar. } \\
\text { Este ejercicio permitió: a) se visibilizar las } \\
\text { narrativas de los docentes como posibles } \\
\text { dispositivos pedagógicos, para orientar la práctica } \\
\text { docente; b) comprender que el quiebre del orden } \\
\text { instituido en la escuela por los estudiantes, no se } \\
\text { debe ver como un problema, sino como } \\
\text { oportunidad para comprenderlos y reorientar el } \\
\text { proceso de enseñanza; y c) la escuela debe ser } \\
\text { repensada constantemente de las necesidades de } \\
\text { sus usuarios más no desde marcos impuestos. En } \\
\text { síntesis, se concluye que la tematización de la } \\
\text { confesión del maestro y la comprensión del } \\
\text { estudiante como texto que reclama ser } \\
\text { comprendido, es un poderoso medio para } \\
\text { repensar la escuela. } \\
\text { Descriptores: Escuela, practica pedagógica, } \\
\text { repensar }\end{array}$ & $\begin{array}{l}\text { This essay aims to show the teaching narration } \\
\text { as possible pedagogical devices to improve } \\
\text { teaching practice. The methodological strategy } \\
\text { was to make a documentary analysis from } \\
\text { some authors who address the issue; this is } \\
\text { accompanied by narratives of teachers in the } \\
\text { school environment. This exercise allowed: a) } \\
\text { the narratives of teachers as possible } \\
\text { pedagogical devices visible to guide teaching } \\
\text { practice; b) understand that the breakdown of } \\
\text { order instituted in school by students, should } \\
\text { not be seen as a problem but as an opportunity } \\
\text { to understand and reorient the teaching } \\
\text { process; and c) the school should be constantly } \\
\text { reconsidered from the needs of its users but not } \\
\text { from imposed frames. In sum, it is concluded } \\
\text { that the theming of teacher confession and } \\
\text { student understanding as text claiming to be } \\
\text { understood, is a powerful means to rethink } \\
\text { school. }\end{array}$ \\
\hline
\end{tabular}

\footnotetext{
* Docente investigador de la Secretaría Departamental de Educación, Antioquia. Licenciado en Filosofía. Magister en Educación Universidad Santo Tomás de Bogotá. Doctorando en Educación Universidad Nacional de Rosario en Argentina. carlosfilosofo@ hotmail.com

** Docente investigadora de la Universidad Cooperativa de Colombia. Profesional en Comercio exterior. Magister en Educación Universidad Santo Tomás de Bogotá. Doctorando en Educación Universidad Nacional de Rosario en Argentina. ingridocente@gmail.com
} 


\section{INTRODUCCIÓN}

Sin duda la escuela es un espacio institucional, pero también simbólico. En esos espacios, se tejen y se entretejen relaciones cruzadas por textos (personas y objetos). En la escuela hay textos por todos lados que debe ser leídos a partir de referentes teórico, para repensar la escuela misma, más allá de su estado instituido, o como diría Bocanegra (2008), más allá de las rejas, de lo banalizado y naturalizado. Precisamente, las confesiones de los maestros como narrativas y la comprensión del estudiante como texto que reclama ser leído y comprendido, son una poderosa herramienta que se puede convertir en posibles dispositivos pedagógicos para orientar la práctica docente, y desde ahí repensar la escuela, dado que esos relatos tienen su arjé en la experiencia real del acto educativo; esto es, experiencia vivida. No se puede olvidar que la vida humana tiene una dimisión narrativa, y es por tal razón que afirma Ricoeur (1996), que toda narración es vivida.

La escuela debe ser repensada desde categorías teóricas, ese es el espíritu del presente ensayó. En él, se propondrá repensar la escuela desde su estado de profanación en la que se encuentran algunos de sus caracteres, a partir de dos categorías:1) confesión del propio texto: conocimiento de sí, tematización de sí, y 2) comprensión del otro como texto que habla: conocimiento del otro, tematización del otro. Esto, tratando de fusionar principalmente los aportes de Foucault (2014) (dispositivos de confesión y conocimiento de sí), y la noción de “texto” de Ricoeur (1996) y Rosas Jiménez (2014)

\section{DESARROLLO}

\section{Mi confesión, julio 20 de 2015}

Empecé mis clases de forma dinámica y noté que todos mis estudiantes reían, porque entre otras cosas suelo comenzar con chistes, o con palabras irónicas o jocosas para romper el hielo. Ese día, traté que los estudiantes tomaran algunas notas textuales de lo dicho en clase; pero no había dictado dos párrafos cuando noté que 4 textos me hablan meneando las piernas de lado a lado, como si me dijeran: No, cuando giraban hacia un lado, y otra vez, no, cuando giraban al lado

Revista EDUCARE, Volumen 19, Número 3, Septiembre -Diciembre 2015. ISSN: 2244-7296Página 156 
opuesto. Eran cuatro chicos que con los movimientos de sus piernas, me decían varías cosas: 1) no estaban interesados la clase; 2) nodeseaban continuar tomando notas; 3) o sencillamente, la temática del día no correspondía a su expectativa, por tanto, no les interesaba mucho el tema ¡Primer campanazo!

En otra clase, también no té que varios textos me hablaban... Mientras explicaba una clase de Filosofía, deslicé mi mirada y observé a una chica pintándose las uñas y contemplando su belleza frente a un espejo de mano. En otro extremo del salón, un chico más, chateaba. Terminó la clase, y para mayor sorpresa cuando llego a la sala de profesores, me doy cuenta que, unos de los estudiantes que me pidió permiso para ir al baño, un compañero profesor lo observó consumiendo sustancias psicoactivas en el baño para hombres ; Segundo campanazo!

Los dos campanazos fueron suficientes para recordarme que: en primer lugar, ya no estamos a otrora, donde se concebía el alumno como un receptáculo pasivo, porque de hecho, la versión neopositivista y si se quiere neotyloriana, llamadas hoy competencias, que objetiva al estudiante, ha cambiado no su molde, pero sí su forma, hasta el punto de permitirle movimiento al alumno, para que sea competente en el mundo de hoy, que tiene caracteres management. Y en segundo lugar, me ubicó en el presente, en el ya y ahora, al recordarme que, la escuela ha sido además de secularizada su matriz por el Estado moderno desde los albores del siglo XVI, y, tal secularización se hace más fuerte en el siglo decimonónico; hoy en la contemporaneidad, la escuela se encuentra profanada. (relato de un profesor,(2015))

Esta confesión no es gratuita, tiene una intencionalidad: repensar la escuela desde su matriz secularizada y profanada ${ }^{\dagger}$, a partir de una concepción del texto como tematización de sí (conocimiento de uno mismo) que presenta Foucault (2012) en los dispositivos de confesión y tematización del otro (conocimiento del otro que expone Ricoeur (1996) y Rosas Jiménez (2014).

$\dagger$ Términos retomado a parir del seminario doctoral en Rosario, Argentina en junio de 2015 por el doctor José Noro, donde brindó pistas para comprender desde una mirada histórica, el nacimiento y constitución de la Escuela.

Revista EDUCARE, Volumen 19, Número 3, Septiembre -Diciembre 2015. ISSN: 2244-7296Página 157 


\section{Confesión del propio texto: conocimiento de sí, tematización de sí}

Al establecer las categorías de confesióncomo conocimiento de sí, y tematización de sí, que automáticamente remite al discurso de Foucault (2014) sobre los dispositivos de confesión relacionados con el decir veraz de si mismo, y el perfeccionamiento del sujeto mediante el conocimiento de si mismo Foucault (2012); la noción de texto de Ricoeur (1996)cuando introduce la categoría del sujeto- texto, que se puede leer para ser comprendido; y Rosas Jiménez (2014) con el paciente como texto, lo que se hace es, retomar este trio categorial para dar a entender que la persona cuando se confiesa es un texto que se conoce a sí mismo, y también se da a conocer a otros, y esto facilita una intervención pedagógica.

Se trata entonces de proponer la confesión de sí, que emana del texto de sí, en busca del conocimiento de sí, pero bajo la tematización de sí, y de esta forma, buscar la transformación de la práctica pedagógica. Algo muy parecido fue tratado por Runge y Muñoz (2012) que también han abordado la tematización de sí pero desde la investigación narrativa, encaminada a mejorar la práctica pedagógica. "Esto con el fin de cruzar la investigación biográfica-narrativa y la formación pedagógica, tendientes a potencializar el campo disciplinar de la pedagogía, y “ buscar un acercamiento a la vida escolar mediante la descripción de experiencias y fenómenos particulares de la cotidianidad que, a pesar de su importancia y su realismo, generalmente no suelen ser registrados y pasan como algo irrelevante para la reflexión pedagógica" (Runge y Muñoz, 2012, p. 6).

Pero obsérvese la diferencia entre lo que proponen estos autores frente a la propuesta que contempla este manuscrito. La preocupación de ellos estriba en potenciar el campo de la pedagogía; mientras la intención de estas líneas, además de potenciar el campo pedagógico mediante la trasformación de la práctica pedagógica, a través de la tematización de sí, y tematización del otro al ser comprendido, es un pretexto para desde ahí repensar la escuela desde el estado en que se encuentra.

Ahora bien, esta trasformación de la práctica pedagógica debe tener presente que, los caracteres no esenciales de la matriz de la escuela (espacio cerrado, frontalidad, verbalización, disciplina, trabajo, horario, distribución del tiempo);sino los accidentales 
(profesor dios del saber, información sólo en los libros, obediencia absoluta del estudiante, seguir un solo relato que conducía a la verdad-conocimiento, entre otras), cambian continuamente, según Noro (2015). También, han cambiado sus actores, por que hay nuevosrelatos, nueva comprensión de las prácticas humanas, que se instauran paulatinamente en la escuela, y cuando tienen raíces y apoyo estatal, se imponen ante los relatos y prácticas que los antecedía para formar nuevos paradigmas.

Estos nuevos paradigmas atraviesan la escuela, y a partir de ahí, se tensionan las prácticas de los sujetos pedagógicos porque hay quienes se quieren ajustar al cambio, al nuevo relato que trae consigo nuevos métodos educativos y formas de educar,y se lanzan al vacio sin paracaídas, porque un nuevo discurso de amor educativo los ha subjetivado, y se dejan encantar por lo que Romero y Romero (2013) llaman la educación Marrana que es aquella que, le gusta el Estado Moderno, porque educa sin tanta inversión económica, presenta métodos y estrategias educativas inéditas que se pueden emplear sin ir a la escuela ( Cine, teatro, televisión, redes sociales, etc.).

Pero pasa algo, cuando estos docentes se dan cuenta que, el discurso amoroso de laeducación Marrana,que pretendía cambiar los males de la educaciónles ha sido infiel,los ha engañado por que sus métodos no resultaron tan efectivos ante la realidad social, no saben qué hacer y se quedansumergidos en la melancolía por la incertidumbre. Siguiendo a González Rios (2014) cuando aborda la metáfora del sueño y la vigilia sería, se podría decir que estos maestros/as, están sumergido en un sueño profundo, donde sólo vive de ilusiones, de apariencias y no puede conocer la realidad como sí la puede conocer el hombre que vive despierto, en vigilia.

Hay otros sujetos pedagógicos (maestros), que no están dispuesto tan fácilmente a dejarse convencer por ésta retórica de amor que propone la educación Marrana, y mantienen posiciones fijas añorando y rumiando el pasado no tan instrumentalizado de la educación. Con éstos, también pasa algo similar, se quedan sólo rumiando de forma melancólica el pasado. A esto es lo que González Ríos (2014) llamó desde una postura filosófica veneración simple del pasado, refiriéndose a aquellas personas que sólo se dedican a repetir lo que por ejemplo, dijo Platón, Aristóteles, Santo Tomas, o cualquier otro pensador, sin interrogar ese pasado para comprender el presente.

Revista EDUCARE, Volumen 19, Número 3, Septiembre -Diciembre 2015. ISSN: 2244-7296Página 159 
Precisamente, ese no interrogar el presente en el acto educativo, es una de las inquietudes que Mosquera (2015a) plantea en su artículo titulado: "Rumiar sobre el positivismo o trasformar la práctica educativa pedagógica docente”, donde critica la postura de los docentes que se quedan rumiando sobre el pasado, o quejándose de los fenómenos educativos que invaden la escuela y no hace nada para transformar la práctica docente. Se debe recordar las entonaciones del pasado desde la escuela, pero con la intencionalidad que permita la relectura de los problemas del presente, para que el pasado permanezca abierto y un presente que le dé cabida a nuevas expectativas, sólo así podemos reinventar la escuela.

Como docentes con años de experiencia pedagógica en el aula, a nuestro juicio, ninguna de las dos posturas: los que se dejan llevar por la "educación marrana", y lo que se oponen al cambio producto de los afanes de la modernidad, son las que corresponden a la realidad de la escuela de hoy; por lo tanto, deben ser repensadas para reinventar la escuela. La salida,no como solución absoluta, dado que no hay salida verdadera, lo que hay son distintas miradas sobre la realidad, no puede ser la actitud melancólica, como si lo es, la transformación de la propia práctica. Y esto se hace yendo al encuentro del problema. Aquí se debe emplear una mentalidad de filósofo, dado que según Gonzales Rios, (2014), el filósofo no le huye al problema, él va a su encuentro, acude a su llamado para poder pensar, precisamente porque no es sabio (sophos), por eso busca comprender de forma incesante los fenómenos de manera provisoria y lo que lo inquieta. Esa es la misma aptitud que se propone para el sujeto pedagógico, pues, las recetas para comprender y actuar en el mundo de la escuela también son transitorias.

Y eso se puede hacer a través del conocimiento de sí que nos propone Foucault, (2012), porque no hay transformación sí no existe un conocimiento previo de sí, para que el sujeto; en este caso, el sujeto pedagógico, se pueda tematizar asimismo. Este conocimiento de sí, que se da gracias al cuidado de sí, en busca de la perfección de sí (Foucault, 2012), es lo que va a posibilitar la transformación de la práctica disciplinar. Eso lo supo Foucault, (2012)al rastrear el conocimiento de sí en su libro "hermenéutica del sujeto", en tres momentos: 1) desde Sócrates y Platón; 2) desde la Filosofía Helenística:Seneca, Epicúreo y estoicos; y 3) Medioevo. 
Respecto al primer momento que leemos a través de Foucault, (2012) en la expresión "conócete a ti mismo" defendido por la ética socrática, que conduce al "ocúpate de ti mismo" épiméleia haute, como una manera de deslizar la mirada sobre sí. Es un estar vigilante para saber guiar el comportamiento, la conducta, el pensamiento para formar el ethos de si mismo, que permite al ciudadano gobernarse así mismo para poder gobernar a lo demás, pues, quien no se gobierne así mismo, tampoco podrá gobernar a otros. Foucault, (2012), también nos habla que Platónpropone algo similar en el sentido que, la razón o alma intelectiva, debe gobernar el alma irascible y concupiscible, y de esta manera el hombre no se pierda en el deseo y apetitos. Esto implica que el alma racional, debe estar buscando su purificación de manera constante a través de la reminiscencia. Esto es una actividad permanente del alma para desprenderse del mundo pecaminoso. Por eso, quien gobierna a los demás debe ser el filósofo porque tiene la sabiduría de ocuparse de sí mismo y de los demás.

Nótese que en el planteamiento que hace Foucault, (2012), ni en Platón ni Sócrates, ocuparse de sí, es encerrase en su mismidad, en el Yo; por el contrario, el ocuparse de sí, es para saberse dirigir, y desde ahí, dirigir y servir sabiamente a la polis-ciudad. Si esto, se analizan bajo una óptica educativa, se puede inferir que, el buen maestro es aquel que se ocupa de si mismo para poder ocuparse de los discípulos. Esa ocupación de sí mismo puede hacerse a través de la preocupación de su propia formación, de su autorreflexión al quehacer diario, y especialmente al ejercicio de autoanálisis acerca de la pasión que le genera el complejo mundo de educar.

En segundo momento observa Foucault, (2012), que existe en todas las escuelas Helenas, una preocupación por el perfeccionamiento constante de sí. Los epicúreos, se confesaban constantemente ante sus miembros con la finalidad de buscar la pureza. Los estoicos, de igual manera se preocupaban tanto de sí, que se desconectaban del mundo superfluo, para concentrarse en descubrir los principios y virtudes mediante la ataraxia, para buscar la perfección de su paz interior y ser libres; tal es el caso de Seneca que, se preocupaba tanto por los actos correctos que debía realizar en el día, que en la noche hacia un inventario de las acciones realizadas, para detectar dónde había fallado. 
En el tercer y último momento Foucault, (2012) también analiza cómo en la Edad Media el monje vivía una vida ascética y cenobítica, regido por los dispositivos de confesión permanente, siempre es busca de la verdad y el conocimiento de lo sagrado, para buscar su perfección.Pero resulta que la vida monacal sobre todo en los siglos IV y V, sostiene Foucault (2012), requería de un guía espiritual ya maduro- el maestro, que tenía un dominio y soberanía sobre si mimo, y por tanto, podía guiar al discípulo a la perfección. Como se puede otear, la noción de épiméleia haute, (preocupación por el conocimiento de sí, introspección de sí para buscar la perfección), ha atravesado la historia y practicada de diferentes maneras. A propósito sostiene Foucault, (2012) que:

"De aquí se derivan toda una serie de prácticas basadas a su vez en toda una serie de ejercicios que van a jugar en la historia de la cultura, de la filosofía, de la moral y de la espiritualidad occidental un papel muy relevante. Entre estas prácticas se encuentran, por ejemplo, la técnica de la meditación, la técnica de la memorización del pasado, la técnica del examen de conciencia, la técnica de la verificación de las representaciones a medidas que éstas se hacen presente en la mente. (pp.37).

Cómo la práctica de la épiméleia haute, ha sido practicada de diferentes maneras en el hilo conductor de la historia de la humanidad, se hace necesario hoy, ante la caída de relatos que marcaban las notas de identidad de la escuela, y los advenimientos de nuevos contra-relatos que la desacralizan, y a veces desactivan al maestro, hasta el punto de no sabe qué hacer porque la perplejidad lo invade; es urgente que el sujeto pedagógico retome la épiméleia hautegriega,pero aplicada a los caracteres de la contemporaneidad. Es decir, se necesita que el maestro se ocupe de sí mismo, para que se conozca, devuelva la mirada crítica sobre su práctica. Luego entonces, examinada su práctica mediante el deslizamiento crítico de su mirada, pueda tematizarla para mejorarla. Por lo tanto, el docente debe confesarse a sí mismo como texto permanente; es como imitar a Seneca, que según Foucault (2012) revisabalo actuado durante el día para buscar el punto que generaba la falla.

Obviamente esto tiene un precio que pagar por el movimiento constate del sujeto pedagógico sobre sí. Toda cualificación para buscar la perfección de la práctica tiene un precio que pagar. Al respecto, explica Foucault (2014) estudiando los dispositivos de confesión del Medioevoque, toda confesión tiene una precio. Por ejemplo, el precio que 
tiene el decir veraz sobre sí mismo, pero que es necesario para buscar la perfección y la salvación del hombre creyente. De igual modo, lo explica Foucault (2012), cuando analiza los estilos de vida de los estóicos, epicúreos en la Filosofía Clásica y la forma de vida ascética de los monjes y religiosos en la Edad Media. Es decir, en estos estilos de vida se debían pagarun precio para buscar la perfección de sí.

El precio que debe pagar el sujeto pedagógico contemporáneo cuando confiesa su texto de si, obviamente que no hace referencia a la vida de ataraxia y ascética, sino un precio en inversión de tiempo, de sacrificio, y si se permite la expresión hasta de mortificación-preocupación, para mejorar de su práctica lo que haya que mejorar. Así, el estudiantado y las personas cercanas al sujeto pedagógico se beneficiarían porque la épiméleia haute griega como ya se dijo, no se reduce a la individualidad, a buscar la perfección de sí, sin un compromiso con la otredad, busca el bien de la colectividad.

Ahora bien, la tematización del texto de sí, no es posible sin establecer una relación con el texto que se lee del otro. Para lograr mayor explicidad partiremos del primer párrafo de mi confesión:

"Ese día traté que los estudiantes tomaran algunas notas textuales de lo dicho en clase; pero no había dictado dos párrafos cuando noté que 4 textos me hablanmeneando las piernas de lado a lado, como si me dijeran: No, cuando giraban hacia un lado, y otra vez, no, cuando giraban al lado opuesto. Eran cuatro chicos que con los movimientos de sus piernas, me decían varías cosas: 1) no estaban interesados en la clase; 2) no deseaban continuar tomando notas; 3 ) o sencillamente, la temática del día no correspondía a su expectativa, por tanto, no les interesaba mucho el tema" (relatos de un profesor op.cit.)

Otéese las tres conclusiones con que cierra la confesión. Estas tres inferencias, se dan porque hay una relación directa del propio texto, con otros textos que hablan. Por consiguiente, es en la premura por comprender la actitud de estos cuatro textos que hablan, que llevan al sujeto pedagógico a tres conclusiones que serán categorías de abordajes, por tanto, serán las que lo subjetiven para la tematización de sí, para la perfección de su practica pedagógica, porque se ha dado cuenta que: A) no ha logrado enamorar a los estudiantes del relato que les vende en la clase; algo está fallando. Las personas caminan, se mueven, se motivan por las clase de relatos que se les ofrezca, y sobre todo, cómo se les

Revista EDUCARE, Volumen 19, Número 3, Septiembre -Diciembre 2015. ISSN: 2244-7296Página 163 
vende; B) toda hermenéutica del otro tiene la intención de comprender el ser a partir de las acciones expresadas en su existencia, intentando recuperar a la vez, el valor óntico de la persona en su actitud propiamente existencial (Rosas Jimenez, 2012). Aquí hay un efecto colateral, la preocupación por el texto del otro, abre los ojos para la cualificación del propio texto; es un cuidado de sí, para cuidar a los demás. Porque pareciera que el valor óntico del los textos que se cruzan en el ámbito escolar, se diluyera a veces ante la vida azarosa de la escuela.

Respecto (A), el sujeto pedagógico en la tematización de sí, debe contemplar también qué relatos debe ayudar a vender para motivar a los textos que lo escuchan en clases. Ya Noro(2015) señala que un caracter de la crisis en la escuela hoy, es la caída de muchos de sus relatos, que dieron origen a la escuela moderna. Luego entonces, si hay caídas de relatos fuertes, el maestro debe instituir otros, no es fácil, pero se debe intentar, porque de otro modo sería muy difícil entonces reinventar la escuela, así sea bajo categoría teóricas. He aquí un elemento más dentro del camino propuesto inicialmente: la confesión de sí, que emana del texto de sí, en busca del conocimiento de sí, pero bajo la tematización de sí; y de esta forma, buscar la transformación de la práctica pedagógica. Es decir, incorporar a la transformación de la práctica pedagógica, la urgencia de nuevos relatos, porque según Noro (2015) son ellos los que ponen en funcionamiento los proyectos, generan convicciones, instalan motivaciones, convencen y acompañan los procesos

En cuanto (B) el sujeto pedagógico necesita recuperar su valor óntico pedagógicamente hablando, para poder ayudar a recuperar al mismo tiempo, el valor óntico de los otros textos que le hablan (estudiantes). Esto bajo la filosofía que dio origen al Movimiento Pedagógico Colombiano (MPC) en los años 70s (Mockus,1984), (Mosquera (2015b). Sería que el maestro recupere su poder, su rol de sujeto de hacedor de la educación, hurtado por las reformas educativas, que instrumentalizaron la educación de los años 60 en toda Latinoamérica, como estrategia hegemónica de la Derecha occidental encabezada por Estados Unidos, para evitar otros posibles Cuba(as) en la Región Latinoamérica teniendo en cuenta la zozobra que generada la post Guerra Fría.

En consecuencia, si el sujeto pedagógico no recupera su poder, su rol mediante la tematización de sí, que le ha sido hurtado por las reformas educativas y otros fenómenos

Revista EDUCARE, Volumen 19, Número 3, Septiembre -Diciembre 2015. ISSN: 2244-7296Página 164 
que se injieren a la educación, y que demás, han creado una especie de subjetividad en él mismo, corre el riesgo de desprofesionalizarse. Se debe aprovechar en cierto sentido la subjetividad quehan producido las reformas educativas en el maestro. Esto último en sentido foucaultiano, pues, sostiene Foucault (2012) que, algunas prácticas y las mismas instituciones donde se instalan las prácticas, subjetivan una especie de sujeto. Sencillamente, si el sujeto pedagógico no recupera su poder, su rol, tendría dificultades para guiarse así mismo, porque sigue encadenado. Por consiguiente, tampoco puede generar relatos fuertes en sus clases que provoquen movilidad en el alumnado.

Si se permite la arbitrariedad, el sujeto pedagógico debe salir y sacar sus prácticas pedagógicas de la Caverna en sentido platónico, para que después de haber perfeccionado sus prácticas mediante el cuidado de sí, y tematización de sí, y teniendo en cuenta las características de cada uno de los personajes que se quedó en la Caverna, se pare en la entrada y empiece a vender relatos fuertes; porque son estos relatos fuertes que harán caminar a los estudiantes hacia la entrada de la caverna y con ello al exterior. Estos relatos además, deben ser pensados como para distintas escuelas, dado que los estudiantes que orientamos son diferentes y tienen distintas necesidades. Son relatos diferentes a como lo ha entendido la racionalidad moderna económica para abaratar costos. Aquí se retoman las palabras de Noro, (2015) al decir que la escuela de los tugurios en sentido de pobreza y de capital cultural, no puede ser la misma de la urbe. Cada escuela debe ser diferente. En este caso, cada relato debe ser diferente, porque cada estudiante es diferente y tiene expectativas diferentes.

\section{Comprensión del otro como texto que habla: conocimiento del otro, tematización} del otro.

Si cada estudiante es un texto, el sujeto pedagógico es un lector que mediante su praxis pedagógica tratará de comprender los textos que se le abren para que ser leídos en la escuela (risas, movimientos corporales, expresiones faciales, inquietudes, la misma habla. etc.), porque "[...] todo lo que es susceptible de ser comprendido puede ser considerado texto: no solamente los escritos mismos, claro está, sino también la acción humana y la 
historia, tanto individual como colectiva, que solo son inteligibles en la medida en que puedan leerse como textos" (Grondin retomado por Jiménez Rosas, 2012, p. 236).

En consecuencia, ya no es bien vista la postura de quienes piensan que tal chico o chica es un problema porque se mueve, muestra apatía a veces en clase, se duerme, habla y pregunta. ¡Sólo es un texto que se está abriendo para que sea leído, por Dios!; Pero además, si se concibiera un problema en sentido filosófico, ¡es magnifico!, porque permite que el sujeto pedagógico, tome la aptitud de filósofo y vaya a su encuentro para asentar su mirada sobre él y comprenderlo. Porque el problema del griego problema (dificultad que sale al encuentro), consiste en que el esfuerzo que haga el filósofo no es para acallarlo, sino más buen acudir a su llamado, ir a su encuentro problemático, porque si hay perplejidad e incertidumbre hay movilidad de pensamiento (González Ríos, 2014). En este caso, también, movilidad de estrategias pedagógicas.

El problema estriba en que el sujeto pedagógico, se ha dejado atropellar por la perplejidad de textos escolares, y no los ve como eso, como textos a interpretar; por el contario, los ve como problemas, problemas de una trama infinita. En rigor, como la pedagogía no está en libros, sino en el cine por las críticas que realiza en las películas como dice Noro (2015), citaremosla escena entre Hilary Swank (Erik Cruwel), profesora de Literatura e Imelda Staunton (Kambell), jefa del Departamento de Literatura,en la película Escritores de Libertad, dirigida por LaGravenese, (2007), respecto al comportamiento de los estudiantes en el Institute Wilson Clssical in Long Beach. En aquella conversación cuando Erik Cruwel llega a la preparatoria a iniciar sus clases, la jefa del Departamento de Literatura cuando revisa el plan de estudios que trae la profesora Cruwel, de entrada le vende la imagen de que los estudiantes son un problema para el Institute Wilson.

Cruwel- Traje mis planes de estudios. Me encantaría que los revisara.

Kambell- Sí. Éstas son las clases con las que iniciarás. Primero de Inglés: cuatro clases de 150 estudiantes de entradas... Muchos acaban de salir del reformatorio; algunos traen artefactos en los tobillos para monitorearlos. Y escucha, revisaré nuestro plan de trabajo, y si ves sus notas, las lecciones de vocabulario y, también estos libros como la Odisea, serán muy difíciles para ellos.

Cruwel- Entiendo.

Kambell- Además muchos toman tres autobuses. Se hacen 90 minutos de viaje.

Cruwel- ¿Tanto así? 
Kambell- Así que yo no les daría demasiada tarea, perderías el tiempo revisando trabajos atrasados.

Cruwel- Claro.

Kambell- Gracias... Que pena que no llegaste aquí hace dos años. Teníamos unas de las clasificaciones más altas del distrito. Desde que la integración voluntaria entró en vigor, hemos perdido más del 75\% de los mejores estudiantes.

Cruwel- Bueno, de hecho elegí Wilson por su programa de integración. Creo que lo que pasa aquí es emocionante... ¿No cree?

Kambell- ... eres una maestra novata. Soy jefe del departamento, debo confiar que eres capaz de lidiar con lo que uno enfrenta aquí (LaGravenese, 2007).

Como se puede observar, a partir de los relatos anteriores, hay una concepción de los estudiantes como problema que le quita prestigio a la preparatoria Wilson para Kambell. Esa no es la aptitud que necesita el sujeto pedagógico; más bien, requiere una aptitud que lo mueva a comprender lo que dicen los textos. La realidad escolar es rica en textos, tiene textos por doquier (estudiantes, maestros, paredes, pupitres, baños, etc.)Allí los textos hablan permanentemente, sólo que no son leídos por el sujeto pedagógico como tal, para poder comprenderlos y tematizarlos. Es necesario entonces, tener una mirada del texto como lo tiene Rosas Jiménez, (2012) de la bioética cuando afirma: “[...] la bioética tiene que centrarse en la persona humana y no perder su mirada sobre ella; tiene que aprender a ver al paciente como un libro abierto que le ofrece múltiples páginas que tienen que ser leídas para dar un dictamen sobre su condición, sobre su salud”' (pp. 236).

Esto es clave. Lo ontológico del texto, debe estar por encima de los aspectos cosméticos de la escuela, porque pareciera que la imagen está por sobre todo. Y como la imagen está por sobre todo, sobre la dignidad de los textos, la escuela sólo se focaliza en lo que la haga visible. Un ejemplo de ello en Colombia es, tratar de quedar bien ante las pruebas censales que mide el conocimiento adquiridos a parir de unos estándares nacionales prescriptos, callando los texto que hablan, por que no se les escucha. Es más, no se les presta atención, por lo tanto, no son tematizados, y desde luego, tampoco comprendidos para ser tematizados, y desde ahí orientarlos, guiarlos, venderles un relato como lo hizo la profesora Erik Cruwel, en la película Escritores de Libertad, configuró unos estudiantes con 
un sentido sobre el mundo y la vida, al subjetivarlos con un relato fuerte, y diferentes al ofrecido por las drogas, las pandillas, la prostitución y el racismo.

Eso es reinventar la escuela desde su estado de profanación en la que se encuentra en la actualidad. Decir que la escuela está profanada no es una exageración, es una realidad. Ya su matriz bañada por la sacralidad en la que nació como práctica monacal no es la misma. Basta analizar las palabras de la señora Kambell, o el segundo párrafo de la confesión que inicia este escrito para darse cuenta de ello. Por eso, se insiste, de nada vale quedarse en la melancolía de la perplejidad del estado de profanación en el que está la escuela. Que su profanación se una excusa para buscar el cambio.

\section{CONCLUSIÓN}

La realidad escolar es un microcosmos donde afloran las eclosiones de fenómenos educativos. Fenómenos que han desacralizado los caracteres accidentales, más no esenciales de la escuela. A eso se le suma que, los grandes relatos que la han acompañado se están resquebrajando a pedazos por el devenir de otros relatos y contra-relatos. En este contexto de situaciones y perplejidad debemos ir al encuentro de esas situaciones complejas, con una mirada de filósofo y pedagogo, para reinventar la escuela desde su estado de profanación. Una mirada que permita el conocimiento de sí y la tematización de sí en busca de la cualificación, de mejorar la práctica; para desde allí ocuparse por comprender otros los textos que hablan y que necesitan ser escuchados, atendidos y guiados.

La escuela requiere docentes que la retroalimenten constantemente, que la imaginen más allá de las rejas, del cerco; como un territorio a explorar donde se trazan mapas pedagógicos todos los días para reinventarla, porque cada rincón y actor de la escuela es un texto que está abierto para ser leído e interpretado. Asimismo, se hace necesario que el maestro como sujeto pedagógico y hacedor de la cultura desintoxique su mente y con ello la práctica pedagógica, alejándose de la racionalidad tecnicista de educación que lo ha enceguecido, vuelto dependiente y reducido a un ejecutor de tareas. El maestro debe volver a ocupar su valor óntico de trabajador de la cultura, pero también, de reinventor del campo

Revista EDUCARE, Volumen 19, Número 3, Septiembre -Diciembre 2015. ISSN: 2244-7296Página 168 
pedagógico. Sólo así, se puede pensar en una escuela con notas de identidad diferente a la que nos mortificada todo los días.

\section{REFERENCIAS}

Bocanegra, M. (2008). Del encierro al paraíso. Imaginarios dominantes en la escuela colombiana contemporánea: una mirada desde las escuelas de Bogotá. Revista Latinoamericana de Ciencias Sociales, Niñez y Juventud, Vol 6, No 1, 219-246.

La Gravenese, R. (Dirección). (2007). Escritores de libertad [Película].

Díaz, J., \& Sarmento, D. (2011). Currículo y prácticas pedagógicas. Voces y miradas con sentido crítico. Bogotá: Universidad de la Salle.

Foucault, M (2014). Obrar mal, decir la verdad: función de la confesión de la Justicia. Curso de Lovain, 1981: Argentina: Siglo veintiuno Editores Argentina S.A

Foucault, M. (2012). Hermenéutica del sujeto, Argentina: Altamira, pp.39

González, J. (2014) ¿Qué es la Filosofía? La potencia de una pregunta imposible; Buenos Aires, Argentina: Biblos.

Mosquera, E. (2015a). Rumiar sobre el positivismo o trasformar la práctica educativa pedagógica docente. Actualidades Pedagógicas, (65), 217-228. Recuperadohttp://revistas.lasalle.edu.co/index.php/ap/article/viewFile/2912/2771

Mosquera, E (2015b). Tres apostes del Movimiento Pedagógico como oráculo del saber”. Revista Educación y Cultura, Núm. 110.

Mockus, A. (1984). Movimiento Pedagógico y defensa de la calidad de la educación Pública . Revista Educación y Cultura, Núm (2)

Noro, J. (2015).La escuela necesita una nueva matriz para recuperar el protagonismo como agencia educativa privilegiada http://es.scribd.com/doc/56580085/05-LA-ESCUELA-DEL-FUTURO-LAESCUELA-NECESITA-UNA-NUEVA-MATRIZ

Ricoeur. P (2009) La vida: un relato en busca de narrador». En Educación y política. De la historia personal a la comunión de libertades. Traducido por Ricardo Ferrara. Buenos Aires: Prometeo Libros

Ricoeur, P. (1996) Sí mismo como otro. Traducido por Antonio Neira (Madrid: Siglo XXI. 
Romero, J., y Romero, P. (2013). La Educación Marrana. Una novela sobre la educación. Infidelidades, celos y melancolía, Santafé, Argentina: Homo Sapiens.

Rosas, C. (2012).El paciente como "texto" según Ricoeur: implicaciones en bioética.Revista Bioética, vol. 22, núm. 2, 2014, pp. 234-240. Recuperado de http://www.scielo.br/pdf/bioet/v22n2/05.pdf

Runge, A. K., y Muñoz, D. A (2012). Los docentes y la tematización de sí: Formación y narración de sí en clave antropocrítica. Medellín: Universidad de Antioquia. 